\title{
Single Nucleotide Polymorphisms, Indels, and Simple Sequence Repeats for Onion Cultivar Identification
}

\author{
Jernej Jakše \\ Department of Horticulture, 1575 Linden Drive, University of Wisconsin, Madison, WI 53706 USA and \\ Biotechnology Faculty, University of Ljubljana, Ljubljana, Slovenia \\ William Martin \\ Agricultural Research Service, USDA, Department of Horticulture, 1575 Linden Drive, University of \\ Wisconsin, Madison, WI 53706
}

John McCallum
Crop \& Food Research, Private Bag 4704, Christchurch, New Zealand

Michael J. Havey

Agricultural Research Service, USDA, Department of Horticulture, 1575 Linden Drive, University of

Wisconsin, Madison, WI 53706

AdDitional InDEX wORDs. Allium cepa, expressed sequence tags, neighbor joining, UPGMA

\begin{abstract}
The commercial production of onion (Allium cepa $\mathrm{L}$.) inbreds, hybrids, and open-pollinated (OP) cultivars would benefit from a robust set of molecular markers that confidently distinguish among elite germplasms. Large-scale DNA sequencing has revealed that single nucleotide polymorphisms (SNPs), short insertion-deletion (indel) events, and simple sequence repeats (SSRs) are relatively abundant classes of codominant DNA markers. We identified 398 SNPs, indels, and SSRs among 35 elite onion ulations and observed that all populations could be distinguished. Phylogenetic analyses of simple-matching and Jaccard's coefficients for SSRs produced essentially identical trees and relationships were consistent with known pedigrees and previous marker evaluations. The SSRs revealed that elite germplasms from specific companies or breeding programs were often closely related. In contrast, phylogenetic analyses of SNPs and indels did not reveal clear relationships among elite onion populations and there was no agreement among trees generated using SNPs and indels vs. SSRs. This discrepancy was likely due to SNPs and indels occurring among amplicons from duplicated regions (paralogs) of the onion genome. Nevertheless, these PCR-based markers will be useful in the quality control of inbred, hybrid, and OP onion seed lots.
\end{abstract}

Commercial onion production is based on open-pollinated and hybrid cultivars. Occasional mistakes at field planting and harvest or mislabeling of seed require grow outs in the appropriate environments to identify specific seed lots. These grow-outs are costly because they can cause significant delays in the movement of seed to market. The onion seed industry would benefit from a robust set of codominant, easily scorable molecular markers for cultivar identification. However onion possesses two main challenges to the development of molecular markers. The first is the huge nuclear genome of onion, more than 36 and 6 times larger than rice (Oryza sativa L.) or maize (Zea mays L.), respectively (Arumuganathan and Earle, 1991). This enormous amount of DNA causes some PCR-based marker systems, such as randomly amplified polymorphic DNAs (RAPDs) and amplified fragment length polymorphisms (AFLPs), to produce too many fragments that may not resolve well in electrophoretic gels (Bradeen and Havey, 1995; Gökçe, 2001). This problem can be reduced by

Received for publication 26 Jan. 2005. Accepted for publication 5 Apr. 2005. We thank Seminis Seed Co. for DNA of doubled haploids of onion. This work was supported by U.S. Dept. of Agriculture, Initiative for Future Agriculture and Food Systems Grant 2001-04434 and five commercial seed companies. Names are necessary to report factually on available data; however, the U.S. Dept. of Agriculture (USDA) neither guarantees nor warrants the standard of the product, and the use of the name by USDA implies no approval of the product to the exclusion of others that may also be suitable.

'Corresponding author. Email: mjhavey@wisc.edu using primers with additional selective nucleotides (Heusden et al., 2000). The second challenge is that onion inbreds and OP populations often retain relatively high levels of heterozygosity (King et al., 1998); hybrids are obviously highly heterozygous. This relatively high heterozygosity with low levels of allelic diversity among onion populations (King et al., 1998) causes molecular markers, especially dominant ones, to appear monomorphic among hybrid or OP cultivars. Codominant restriction fragment length polymorphisms (RFLPs) exist among elite onion populations (Bark and Havey, 1995; King et al., 1998), but their identification is too expensive and time-consuming to routinely use for cultivar identification. Although codominant SSRs have been identified in onion (Fischer and Bachmann, 2000), relatively complex PCR reaction conditions were difficult to use.

Large-scale sequencing of expressed sequences and genomic regions in onion revealed that SNPs and SSRs are relatively abundant classes of codominant DNA markers (Kuhl et al., 2004). SNPs are polymorphic nucleotides at orthologous positions in the genome. SSRs exist as different numbers of short nucleotide repeats. In this report, we identified SNPs, small insertions or deletions (indels), and SSRs in genomic regions highly similar to expressed sequence tags (ESTs) of onion (Kuhl et al., 2004; Martin et al., in press) and assessed their usefulness to distinguish among elite onion inbreds and populations. 


\section{Materials and Methods}

We evaluated 25 commercial populations, five each from five seed companies [European company A (A1 to A5), European company B (B1 to B5), a U.S. dehydrating company (D1 to D5), a U.S. company (US1 to US5), and a Japanese company (J1 to J5)], and inbreds from the USDA (B1794B, B1828B, B7728B, MSU826B, MSU611-1B, MSU2399B, and MSU8155B), Wisconsin (W202B and W420B), and Polish (P) onion-breeding programs. These populations were classified by the individual companies or breeding programs according to their day-length response for bulbing [long (LD), intermediate (ID), vs. short (SD) day] and their relative storage abilities. Populations A1 through A5; B2, B3, and B5; J1 through J5; US1 and US2; and the USDA, UW, and Polish inbreds were classified as LD with relatively long storage ability. A1 through A5, B2, and B3 are European Rijnsburger types. US3 is a LD Spanish type (grown primarily in the northwestern United States) population with relatively short storage ability. B1 and US5 are short-day and B4 and US4 are ID populations with relatively poor to intermediate storage abilities. The dehydrating populations were classified as SD (D1), LD (D5), or ID (D2, D3, and D4). USDA inbreds $\mathrm{B} 1794 \mathrm{~B}$ and $\mathrm{B} 1828 \mathrm{~B}$ were included because their pedigrees were reported in release notices and they trace back to crosses between a pair of publicly developed onion inbreds. B1794B was released as an $\mathrm{F}_{3} \mathrm{M}_{5}$ population from the cross of MSU8155B with MSU826B ( $M=$ massing generation in which three to five full-sib plants are intercrossed; $\mathrm{F}$ or $\mathrm{S}=$ self pollination of single plant). B1828B was reported as an $\mathrm{F}_{2} \mathrm{M}_{2} \mathrm{SM}_{3}$ population from the cross of MSU826B with MSU611-1B. DNAs were isolated from at least 50 seedlings of each population by CsCl-banding as previously described (Bark and Havey, 1995). Ten onion ESTs were selected that carried genetically mapped SNPs and indels in genomic amplicons revealed using nested oligonucleotide primers (Table 1) (Kuhl et al., 2004; Martin et al., in press). PCR conditions were described by Martin et al. (in press). PCR reactions were run on $1 \%$-agarose gels, stained with $\mathrm{EtBr}$, and single amplicons from each onion accession were cut out of the gel, purified through columns (Qiagen, Valencia, Calif.), ligated into the pGEM-T vector (Promega, Madison, Wis.), and transformed into competent bacteria following the manufacturers' protocols. At least five individual colonies from each gel-isolated fragment were sequenced as previously described (Lilly and Havey, 2001). Sequences were aligned using Sequencher (Genecodes, Ann Arbor, Mich.) to reveal SNPs and indels. We required SNPs or indels to be present in at least two amplicons from at least two different onion populations to avoid scoring amplification or sequencing errors as polymorphisms. The 10 onion cDNAs carrying SNPs or indels were hybridized to DNA-gel blots (Bark and Havey, 1995) of onion doubled haploid DNA (gift of Seminis Seed Co., Woodland, Calif.) to estimate relative copy numbers. For SSRs, onion ESTs carrying SSR-like regions were selected using the MISA program (Thiel et al., 2003) and primer pairs designed as described by Kuhl et al. (2004). Forward primer from each pair was labelled fluorescently $\left(5^{\prime} \mathrm{FAM}\right)$ or one primer from each pair was tailed with the ABI-forward sequence 5'-GACGTTGTAAAACGACGGCC (Xin et al., 2004) and a universal fluorescently labelled primer ( $\left.5^{\prime} \mathrm{FAM}\right)$ of the same tail sequence was synthesized (Table 2). PCR conditions with the two primers were an initial denaturation at $95{ }^{\circ} \mathrm{C}$ for 4 min followed by 28 cycles at $94{ }^{\circ} \mathrm{C}$ for $30 \mathrm{~s}, 58^{\circ} \mathrm{C}$ for $45 \mathrm{~s}$, and $72{ }^{\circ} \mathrm{C}$ for $1 \min 20$ $\mathrm{s}$, and the final elongation step was at $72{ }^{\circ} \mathrm{C}$ for $8 \mathrm{~min}$. The threeprimer PCR protocol is described by Schuelke (2000), timing conditions were the same as for two primer protocol except that annealing temperature was lowered to $55^{\circ} \mathrm{C}$ and 35 cycles were completed. PCR reactions were diluted with 4 -fold with water, $3 \mu \mathrm{L}$ of reaction was mixed with $10 \mu \mathrm{L}$ of formamide and ROXlabeled molecular marker(Chimerx, Milwaukee). Fragments were

Table 1. Sequences of nested oligonucleotide primers producing single amplicons carrying polymorphic indels or single nucleotide polymorphisms among elite onion populations.

\begin{tabular}{|c|c|c|c|c|}
\hline \multirow[b]{2}{*}{ Onion ESTy } & \multicolumn{2}{|c|}{ Primer sequences $\mathrm{z}$} & \multirow[b]{2}{*}{ Inside } & \multirow{2}{*}{$\begin{array}{l}\text { Amplicon } \\
\text { size (bp) }\end{array}$} \\
\hline & Dir. ${ }^{x}$ & Outside & & \\
\hline \multirow[t]{2}{*}{ ACAAJ79 } & $\mathrm{F}$ & CGCATAGAACTGACACCACTTTTCCTCG & TGACACCACTTTTCCTCGTTTG & 682 \\
\hline & $\mathrm{R}$ & TCCAGGAAGGATCAATGTCACACAAAAT & CTTAAAGTCTTCTTCGCTTGGC & \\
\hline \multirow[t]{2}{*}{$\mathrm{ACABX} 01$} & $\mathrm{~F}$ & CTGCTGAGTGCATACATCATCAAAAAAC & CATTCTCACAGTGTTCTTTTGCGG & 495 \\
\hline & $\mathrm{R}$ & GATACAGCACCTCTCATCAGACCAGCCC & ATTGGATAAGAAAGAAAGAGGGAA & \\
\hline \multirow[t]{2}{*}{ ACACU34 } & $\mathrm{R}$ & GAACCAGGTGGTCCATATAGAAGTATGC & CСАСТТСАТССААССТАТСТТСАТСС & 854 \\
\hline & $\mathrm{F}$ & GAAGAACCTAAGATTAGGATGAATAAGG & GTCAGACTTGGTGATGTTGTATCTGTCC & \\
\hline \multirow[t]{2}{*}{ ACADT92 } & $\mathrm{F}$ & CCCAGATATTTGAGCTTTGTAAAAGGTG & ATGATCTACCCCTCAATGTTTCTCGTGA & 632 \\
\hline & $\mathrm{R}$ & CTCTGGCATATTTTCAACATATTGATCC & GTGGTTCCCAGAATCCTCAATGCCA & \\
\hline \multirow[t]{2}{*}{ ACAED83 } & $\mathrm{F}$ & TTTGGTGATGCTAGTACCGAAGACTCTG & AAGATTACGTCCACGTTCGCATAC & 596 \\
\hline & $\mathrm{R}$ & ACCCTAAGCCTAGTACATGCCATGATTT & GTTCTTCCTCTGATCACCTTGCAG & \\
\hline \multirow[t]{2}{*}{ ACADJ12/ACAFK13 } & $\mathrm{F}$ & AAAGATTGGGCTGTTTGGTGGTGCTG & TTCTCTGTCTTTGCTGGAGTTGGTGA & 598 \\
\hline & $\mathrm{R}$ & ATGGAAAGGCTGGCTCAAGAAACGCT & TGTCAAATCGTCAGCAGGCACATAAA & \\
\hline \multirow[t]{2}{*}{ ACAAX07 } & $\mathrm{F}$ & GGGACGACATCGCCCCTGTACATGAG & TGAGTTGGTGATTTCAGCCACGGAGA & 394 \\
\hline & $\mathrm{R}$ & TCTAGAGCACACCGATGTCTCCGTC & GCATGTGCCCCGTGCTATATTTGTTG & \\
\hline \multirow[t]{2}{*}{ ACAET81 } & $\mathrm{F}$ & GAAGGAACTCCGGTTCTAGTGCATG & TGCTAACATTGCACATGGGAACTCTT & 436 \\
\hline & $\mathrm{R}$ & ATCATGCTCATGGTGGAAAAGGAGC & ACAACAGCATCAAAAGCACCAGCAG & \\
\hline \multirow[t]{2}{*}{ ACAGT43/ACAIC61 } & $\mathrm{F}$ & GAGTATGTTAAGGCCATCAAGGAGG & TATCGATGTTTTGGTCCATGGAGAG & 689 \\
\hline & $\mathrm{R}$ & AGCGGGAGTTCTCAATTGTGATCAC & GTCGATGATTGAGTGGATGATGTCG & \\
\hline \multirow[t]{2}{*}{ ACAHO42 } & $\mathrm{F}$ & GGTTAGAATCACGAGGTCATAAATACAT & GAGAAACACATTCCAGACTTGC & 746 \\
\hline & $\mathrm{R}$ & AACCATTCAACTGTAATCTTCTTTATCA & ATGGTTTCAAACGCTGAAGTAA & \\
\hline
\end{tabular}

zPrimer sequences were reported by Martin et al. (in press) and are included here for convenience only.

${ }^{\mathrm{y}} \mathrm{EST}=$ expressed sequence tag.

${ }^{x}$ Direction listed as forward $(\mathrm{F})$ and reverse $(\mathrm{R})$. 
separated on an automatic sequencing machine (ABI3700) and revealed using the Genescan (ABI, Foster City, Calif.) software. A total of 398 polymorphisms were revealed among the 35 onion DNAs. All polymorphisms were scored as presence (1) vs. absence (0) of a specific state. NTSYS-pc version 2.02i (Exeter Software, Setauket, N.Y.) was used to calculate similarity matrices using the simple-matching (the presence and absence of fragments in two populations are both scored as matches) and Jaccard's (only presence of fragments in two populations scored as matches) coefficients. Relationships among populations were assessed using NTSYS-pc and the unweighted pair group method with arithmatic mean (UPGMA) and neighbor joining (NJ) analyses (Rohlf, 1993).

\section{Results and Discussion}

We evaluated 35 elite commercial and public onion populations for 10 genomic regions carrying SNPs or indels and for 58 SSRs to reveal totals of 327 SNPs, 34 indels, and 37 SSRs among the onion populations (Tables 1 and 2). We observed that all populations could be distinguished. Both UPGMA and NJ analyses of simple-matching and Jaccard's coefficients for SSRs produced essentially identical trees (Fig. 1) and estimates of genetic relationships were consistent with known pedigrees and previous marker evaluations. Using RFLPs, we previously demonstrated that the LD storage onions are a derived group relative to onion populations grown under shorter days (Bark and Havey, 1995). Phylogenetic analyses of SSR markers were consistent with a similar genetic background for U.S. and Japanese LD storage populations, consistent with the introduction of storage germplasm from the United States to Japan (Goldman et al., 2000). In addition, these markers revealed that elite germplasms from specific companies or breeding programs were often closely related, likely due to the company's predominance in a specific market class. Publicly released USDA inbreds were all closely related (Fig. 1). European LD storage populations were also closely related, but distinct from U.S. and Japanese storage populations. US3, a Spanish-type population, was placed more closely to LD storage than ID or SD populations. ID and LD populations of dehydrator onions were closely related, but clearly separated from D1, a SD dehydrator population. ID and SD populations with relatively short storage abilities comprised the last well defined group of germplasms based on SSRs.

The SNP markers did not reveal clear relationships among elite onion populations and there was no agreement among trees generated using SNPs and SSRs (figure not shown). We compared trees based on SNPs or indels in exons vs. introns and observed no greater resolution of relationships among the elite populations. This discrepancy between relationships estimated using SSRs and SNPs/indels could be due to the amplification of genomic fragments from duplicated regions of the onion genome (paralogs). We selected onion ESTs showing significant similarities to single positions in the rice genome and designed nested PCR primers to amplify from these regions (Kuhl et al., 2004). In the segregating family from BYG15-23 × AC43, single genomic amplicons were produced by the nested primer sets and SNPs or indels in these genomic amplicons segregated as single loci (Martin et al., in press). In this study, we also observed single amplicons from the onion populations; however sequencing of these genomic amplicons revealed numerous SNPs and indels not observed in

Table 2. Sequences of oligonucleotide primers and nature of repeats for polymorphic simple sequence repeats identified among elite onion populations.

\begin{tabular}{|c|c|c|c|c|c|}
\hline $\mathrm{SSR}^{z}$ & $\begin{array}{c}\text { Fragment } \\
\text { sizes } \\
(\mathrm{bp}) \\
\end{array}$ & Onion ESTy & Forwardw & Reversew $^{w}$ & Type of repeat \\
\hline ACM004 & $\begin{array}{l}203,206 \\
213\end{array}$ & ACS31-JM4.F01 & TCGTTCTTTAGAACACGTTAGGAA & TGTCGGCGGATATAGTGACA & $(\mathrm{CAA}) 4$ \\
\hline ACM006 & $\begin{array}{l}220,223 \\
226,232\end{array}$ & ACB62-JM6.C10 & GCAGTTCTCCCTTTGTAAAATCA & GTGATGGATGAGTGGATGGA $^{\mathrm{w}}$ & (CTC)7 \\
\hline ACM013 & $\begin{array}{l}183,186 \\
192\end{array}$ & ACAHX21 & CAACCTCGAAGAACTCACCG ${ }^{\mathrm{w}}$ & GCGAATCTTGTTTTTGGGAA & $(\mathrm{TCC}) 9$ \\
\hline ACM017 & 265,268 & ACAAM70 & ССТТСТССССАТТСТСТТСС ${ }^{w}$ & CATCGTCCTCGTCCTCATC & $(\mathrm{CAT}) 5$ \\
\hline ACM018 & 275,278 & ACAAN60 & GGGGAATGGTGGAGAATAGA & AACAGAGGCAAGAGGAGCG & $(\mathrm{CTT}) 6$ \\
\hline ACM024 & $\begin{array}{l}120,123 \\
126,131 \\
134,137, \\
140\end{array}$ & ACABS25 & ССССАТТТТСТТСАТТТТСТСА & TGCTGTTGCTGTTGTTGTTG & (GCA) $10-\mathrm{NNN}-(\mathrm{GCA}) 4(\mathrm{ACA}) 4$ \\
\hline ACM031 & $\begin{array}{l}205,207 \\
209\end{array}$ & ACAAD80 & CCAAAGCCGACCTCCTCT ${ }^{\mathrm{w}}$ & CGTGGGAAGACCAAGGGT & $(\mathrm{AC}) 6$ \\
\hline ACM045 & $\begin{array}{l}264,267 \\
269,271\end{array}$ & ACAAW78 & AAAACGAAGCAACAAACAAAA & CGACGAAGGTCATAAGTAGGC $^{w}$ & (TC) 6 \\
\hline ACM066 & 184,186 & ACADR 40 & CTCCCCGCAACCAGTAATAA & GCTTGGGTTTTGTTTCTCCA & (TA) $5-\mathrm{NNN}-(\mathrm{CT}) 7$ \\
\hline ACM068 & 277,281 & ACADD43 & CGAAGGTGAAGGTGTACGGT $^{\mathrm{w}}$ & CAAATGGCTGCAATAAGCAA & (TA) 6 \\
\hline ACM071 & $\begin{array}{l}162,166 \\
167,170\end{array}$ & $\mathrm{ACABO} 63$ & TCTCATTTCAACTTTCTACCTATCC & CTGACATTTGCTCGACTGGA $^{\mathrm{w}}$ & $(\mathrm{AG}) 10$ \\
\hline ACM078 & 282,288 & ACACJ44 & CGCAGAATCTCGTCCTTTTTw & AATGGTTTGGAGGTCAGTCG & (TCG)7 \\
\hline
\end{tabular}


Table 2. Continued.

\begin{tabular}{|c|c|c|c|c|c|}
\hline $\mathrm{SSR}^{z}$ & $\begin{array}{l}\text { Fragment } \\
\text { sizes } \\
\text { (bp) }\end{array}$ & Onion ESTy & Forward $\mathrm{x}$ & Reverse $^{x}$ & Type of repeat \\
\hline ACM082 & $\begin{array}{l}200,203 \\
206,209\end{array}$ & ACACY23 & CACCGTTCCTCAGCTCACTT & AGAGGGACGAAATGAAAGCA & $(\mathrm{TCT}) 13$ \\
\hline ACM091 & 177,183 & ACAFQ28 & TCTCCTCСТCTAACCAGCCA & GGTGCTCCAGTTGAGCTTTC & $(\mathrm{TCT}) 10$ \\
\hline ACM093 & 134,137 & ACAHV10 & GCCAACAGTTTTCGTAAGTTGA & ATTCTCTTCGGCTTTCGTGA & $(\mathrm{CCA}) 7$ \\
\hline ACM094 & 110,114 & ACABX11 & GATGATGGCGAAGACACAGA & AAAAACGGCTTAGGAATTTAACG & (TGG)5 \\
\hline ACM097 & 194,197 & ACAHU83 & TTCTCAGCTGCAATGGTGAC & ACGTGCTTGGGCATTATTTC & (AAG)4 \\
\hline ACM099 & 183,186 & ACAHR66 & CATTTCTCCCTTCTGCTTTGTT & TTTGGATTGTGAGTTTGGCA & (ATC)6 \\
\hline ACM101 & $\begin{array}{l}209,212 \\
215,221\end{array}$ & ACAHO88 & CCTTTGCTAACCAAATCCGA & CTTGTTGAGAAGGAGGACGC & (TCC) 5 \\
\hline ACM102 & $\begin{array}{l}151,154 \\
160\end{array}$ & ACAHL43 & TGGATTTGTGAACAACCGAA & GATGCAGGCAGTGTTTTGAA & $(\mathrm{CAA}) 7$ \\
\hline ACM105 & 151,154 & ACAGL15 & CAAGTGGAGCGGGTATTTGT & GAGGCACAACTTCCTCTTCG & $(\mathrm{ATG}) 5$ \\
\hline ACM112 & 177,180 & ACADA50 & TTCCCAACAAACGTTCATCA & GTGAAGGGAGAGCAGTGGAG & (TCC) 5 \\
\hline ACM115 & 239,242 & ACAGH25 & TCCATCTATGCATCTGCCAC & CTATTCTTCCACTGGGGCAA ${ }^{w}$ & $(\mathrm{CAC}) 6$ \\
\hline ACM119 & $\begin{array}{l}252,255 \\
261,265\end{array}$ & $\mathrm{ACAFC0} 4$ & TTTCAGCAACATAGTATTGCGTC & TCTTCGGGATTGGTATGGAG ${ }^{\mathrm{w}}$ & $(\mathrm{AAT}) 8$ \\
\hline ACM121 & $\begin{array}{l}163,166 \\
172\end{array}$ & ACAFQ44 & GCAAACTCATATAGTGCCGC ${ }^{w}$ & GAACCGATTCTACGAGCAGC & $(\mathrm{TAT}) 5$ \\
\hline ACM124 & $\begin{array}{l}220,228 \\
245\end{array}$ & ACAAB30 & GCAAACTTGAATCCCTTCCA ${ }^{w}$ & AACCCGTTAAGAGGAGGGAA & $(\mathrm{AAAG}) 5$ \\
\hline ACM132 & $\begin{array}{l}200,202, \\
204,206, \\
208,212, \\
220,224, \\
228\end{array}$ & ACABH94 & ATGGGGCCTGGTAAGTTTTT & TGCACACCGTTTCCATTTTA & $(\mathrm{ACAT}) 14 \mathrm{AC}(\mathrm{CATG}) 4$ \\
\hline ACM133 & $\begin{array}{l}191,193 \\
196,198\end{array}$ & ACACL08 & CCACATGGATGAAAAACACAA ${ }^{w}$ & CGCTGGTAGCTGAAGCAAAT & (CA)8(CG)6 \\
\hline ACM134 & $\begin{array}{l}192,200 \\
201,204\end{array}$ & ACACT16 & ACACACACAAGAGGGAAGGG & CACACACCCACACACATCAA $^{w}$ & $(\mathrm{GA}) 8$ \\
\hline ACM138 & $\begin{array}{l}235,239 \\
247,264 \\
268\end{array}$ & ACAEM68 & ACGGTTTGATGCACAAGATG & CCAACCAACAGTTGATACTGC $^{\mathrm{w}}$ & $(\mathrm{CTGC}) 11$ \\
\hline ACM146 & 213,216 & ACAHX41 & ATGTCCCAATTCGACCAGAG & CGTTACGGCTGAGAACTTCC & $(\mathrm{ACA}) 5$ \\
\hline ACM147 & 264,267 & ACAIE32 & CACTTTCCCGTCTAATCGACA ${ }^{w}$ & TTCCCACAATCAAAACACCA & $(\mathrm{CTC}) 5$ \\
\hline ACM151 & 264,266 & ACAGO25 & TGTCAGACAAGCAACTCCTCC & AGGTGAGGCTTAGATGGGGT ${ }^{\mathrm{w}}$ & $(\mathrm{ACA}) 5$ \\
\hline ACM152 & $\begin{array}{l}229,241 \\
244\end{array}$ & ACAFD18 & TCCCAAGAGTCCAAGAATGG ${ }^{\mathrm{w}}$ & TGTTCTCCCTTAAACAGTGCAA & $(\mathrm{CAG}) 8$ \\
\hline ACM169 & $\begin{array}{l}259,261 \\
267\end{array}$ & ACADN53 & ACTTTCCСССТCCAACATTC ${ }^{w}$ & TAGCACAAGGAGGGTCGAGT & $(\mathrm{TA}) 30$ \\
\hline ACM170 & 227,236 & ACADV 27 & TTCTGCAATGAAAACACATTGA & ATCCAACTGAGTCGGCAATC ${ }^{w}$ & (TTC)6 \\
\hline ACM187 & $\begin{array}{l}228,260 \\
262,264\end{array}$ & ACAHZ42 & GTACTCGGGCAGTGGAGGTA & GGAGCTGTCCAAATGCTAGG $^{\mathbf{w}}$ & (GT)6 \\
\hline
\end{tabular}




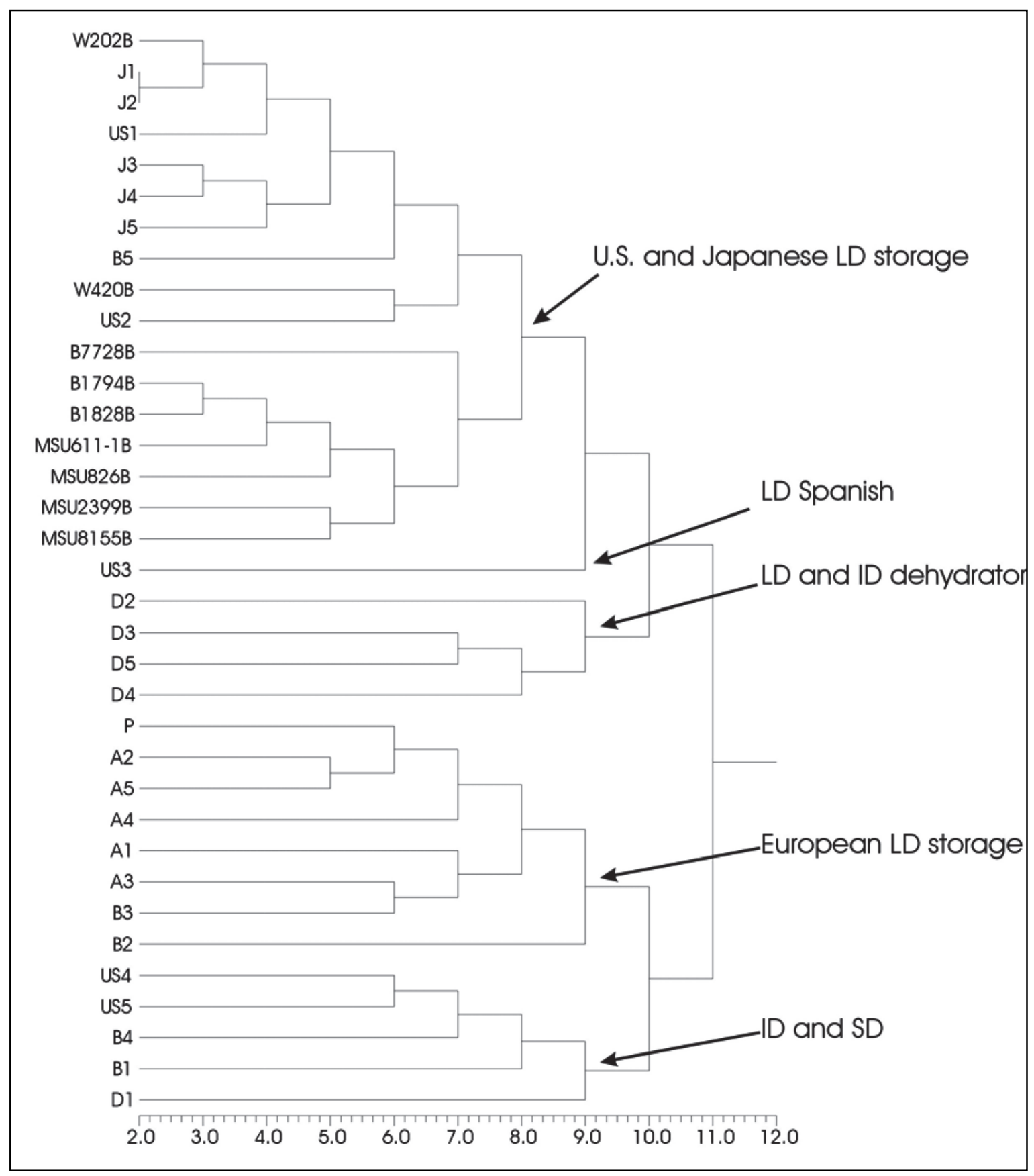

Fig. 1. Neighbor joining analyses of Jaccard's similarities for simple sequence repeats among populations of onion (listed on left). Designations of major nodes are shown with arrows on right. LD, ID, and SD indicate long-day, intermediate-day, and short-day response for bulbing, respectively. Spanish refers to U.S. populations of Spanish-type onions.

the BYG15-23 × AC43 family, which would be expected if the elite populations were more genetically diverse than BYG15-23 and AC43 are to each other. We hybridized the onion cDNAs revealing SNPs and indels to double haploid DNA of onion and observed that some ESTs were duplicated in the onion genome (Fig. 2). As a result, amplicons produced from duplicated regions (paralogs) may accumulate SNPs or indels at different rates and this sequence variability did not reveal relationships consistent with other marker classes, such as RFLPs or SSRs. In contrast, the SSRs were located within coding regions and often were allelic (Martin et al., in press).

The utility of these molecular markers for quality control of onion seed production can be illustrated using the USDA inbreds $\mathrm{B} 1794 \mathrm{~B}$ and $\mathrm{B} 1828 \mathrm{~B}$. Inbred B1794B was reported to be an $\mathrm{F}_{3} \mathrm{M}_{5}$ population from the cross of MSU8155B with MSU826B. Inbred B1828B was reported as an $\mathrm{F}_{2} \mathrm{M}_{2} \mathrm{SM}_{3}$ population from the cross of MSU826B with MSU611-1B. We compared the genotypes of the parental and progeny inbreds to see if any polymorphisms were present in the progeny inbreds that were lacking on both parents. For B1794B, all fragments present in this inbred were also present in one or both of the parental inbreds. However for B1828B, we observed six SSR fragments [ACM024 (134 and 137 bp), ACM071 (162 bp), ACM082 (209 bp), ACM134 (200 bp), and ACM151 (264 bp)] present in this inbred that were 


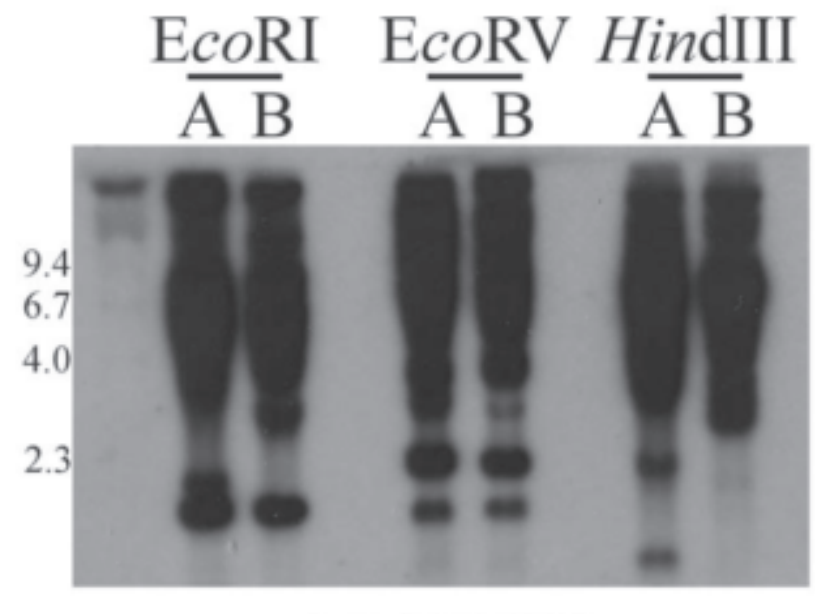

ACABX04

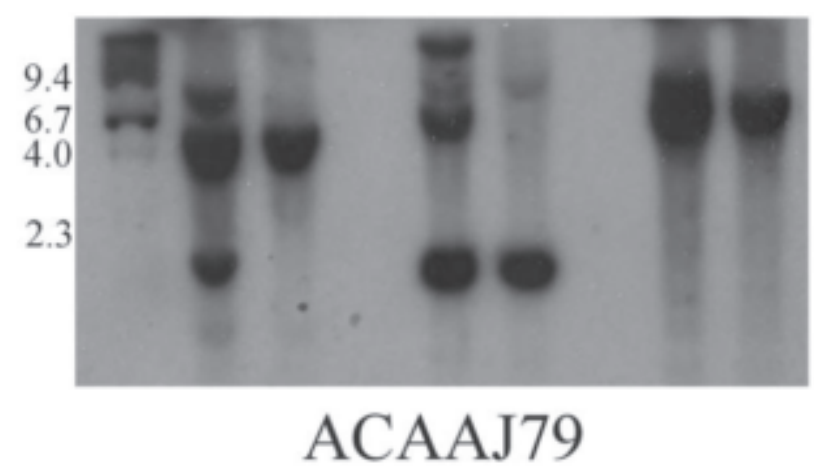

Fig. 2. Representative autoradiograms revealing relative copy numbers of genomic amplicons of onion carrying SNPs or indels, corresponding to onion expressed sequence tags ACAAJ79 and ACABX04. DNAs were from two (A and B) doubled haploid populations of onion. Restriction enzymes are listed above the DNA names. The left-most lane is the size marker with sizes of fragments in kilobases shown on left.

absent from both parental inbreds. The most likely explanation is that the pedigree of B1828B was not correctly reported in the release notice.

It is our intention that these PCR-based SSR, SNP, and indel markers aid in the quality control of inbred and hybrid seed lots. However we caution about use of these markers to demonstrate identity among specific inbreds or hybrids. Even if two populations were identical for all markers developed in this study, one could always argue that evaluation of additional markers may reveal a polymorphism.

\section{Literature Cited}

Arumuganathan, K. and E. Earle. 1991. Nuclear DNA content of some important plant species. Plant Mol. Biol. Rpt. 9:208-218.

Bark, O.H. and M.J. Havey. 1995. Similarities and relationships among open-pollinated populations of the bulb onion as estimated by nuclear RFLPs. Theor. Appl. Genet. 90:607-614.

Bradeen, J.M. and M.J. Havey. 1995. Randomly amplified polymorphic DNA in bulb onion and its use to assess inbred integrity. J. Amer. Soc. Hort. Sci. 120:752-758.

Fischer, D. and K. Bachmann. 2000. Onion microsatellites for germplasm analysis and their use in assessing intra- and interspecific relatedness within the subgenus Rhizirideum. Theor. Appl. Genet. 101:153-164.

Gökçe, A.F. 2001. Molecular tagging of male fertility restoration locus and its selection in onion (Allium cepa L.). PhD Diss., Univ. of Wisconsin, Madison.

Goldman, I.L., G. Schroeck, and M.J. Havey. 2000. History of public onion breeding programs and pedigree of public onion germplasm releases in the United States. Plant Breeding Rev. 20:67-103.

Heusden, A.W. van, M. Shigyo, Y. Tashiro, R. Vrielink van Ginkel, and C. Kik. 2000. AFLP linkage group assignment to the chromosomes of Allium cepa L. via monosomic addition lines. Theor. Appl. Genet. 100:480-486.

King, J.J., J.M. Bradeen, and M.J. Havey. 1998. Variability for RFLPs and relationships among elite commercial inbred and virtual hybrid populations of onion. J. Amer. Soc. Hort. Sci. 123:1034-1037.

Kuhl, J.C., F. Cheung, Q. Yuan, W. Martin, Y. Zewdie, J. McCallum, A. Catanach, P. Rutherford, K.C. Sink, M. Jenderek, J.P. Prince, C.D. Town, and M.J. Havey. 2004. Aunique set of 11,008 onion (Allium cepa) ESTs reveals expressed sequence and genomic differences between monocot orders Asparagales and Poales. Plant Cell 16:114-125.

Lilly, J.W. and M.J. Havey. 2001. Short repetitive motifs contributed significantly to the huge mitochondrial genome of cucumber. Genetics 159:317-328.

Martin, W., J. McCallum, M. Shigyo, J. Jakse, J.C. Kuhl, N. Yamane, K.C. Sink, C.D. Town, and M.J. Havey. 2005. Genetic mapping of expressed sequences in onion and in silico comparisons show scant collinearity with rice. Mol. Genet. Genomics (In Press).

Rohlf, F.J. 1993. NTSYS-pc: Numerical taxonomy and multivariate analysis system. Exeter Software, Setauket, New York.

Schuelke, M. 2000. An economic method for the fluorescent labeling of PCR fragments: A poor man's approach to genotyping for research and high-throughput diagnostics. Nature Biotech. 18:233-234.

Thiel, T., W. Michalek, R.K. Varshney, and A. Graner. 2003. Exploiting EST databases for the development and characterization of genederived SSR-markers in barley (Hordeum vulgare L.). Theor. Appl. Genet. 106:411-422.

Xin, Z., J. Chen, and J. Burke. 2004. A low cost high efficient method for genetic mapping using indel markers. Poster abstract 227. Plant and Animal Genomes 12 Conf., San Diego, Calif. 22 Feb. 2005. <http:// www.intl-pag.org/12/abstracts/P3b_PAG12_227.html>. 